\title{
Reseña del libro Acerca de la apropiación de tecnologías: teoría, estudios y debates Apropiaciones tecnológicas: ¿Cómo imaginar un mundo tecnodigital diferente?
}

\author{
Technological Appropriations: How to imagine a different technodigital world? \\ Review of the book About technology appropriation: theory, studies and debates
}

DOI: https://doi.org/10.22235/d.v0i30.1803

\author{
Mauricio Nihil Olivera
}

Mauricio Nihil Olivera Universidad de la República,

Montevideo, Uruguay.

http://orcid.org/00000003-0018-1940 mauricio.olivera@fic.edu.uy

Recepción: 22/02/2019 Aceptación: 25/03/2019
La perspectiva de la apropiación de las tecnologías (TIC) se ha desarrollado en los últimos años y sostiene la idea de apropiación como un espacio pertinente de observación para comprender relaciones y tipos de vínculos que se establecen con las tecnologías digitales en el ámbito de la interacción y la información, sobre todo, tanto desde el punto de vista individual como grupal, institucional y comunitario. El libro Acerca de la apropiación de tecnologías: teoría, estudios y debates (Lago Martínez, Álvarez, Gendler y Méndez, 2018) presenta una puesta a punto del avance y estado de la discusión sobre ese desarrollo. Cabe señalar, aunque pueda no ser un dato desconocido para quienes se acerquen a este libro, que varios de los autores tienen una reconocida producción y una importante acumulación de conocimiento sobre estas temáticas.

De este modo, se constituye en un texto de referencia para conocer el estado del arte actual de la perspectiva de la apropiación de las tecnologías, así como para quienes deseen abordar esta perspectiva desde diversas dimensiones: el análisis y evaluación de la brecha digital; las habilidades y competencias tecnológicas de quienes las usan; la importancia que los contextos culturales, sociales y económicos desiguales tienen sobre la apropiación; las políticas públicas de inclusión digital y la evaluación de sus resultados en términos de reducción de la brecha; el rol del mercado en las prácticas de apropiación y las innovaciones emergentes.

En todos los casos está presente la idea de apropiación como orientadora de procesos de investigación y análisis que abarcan desde cuestiones más generales -la comprensión de distintas dimensiones de la sociedad y la cultura en que vivimos- hasta problemas más específicos relativos a políticas públicas, aspectos institucionales y culturales, así como estrategias para el trabajo con tecnologías en el aula.

El libro se divide en cuatro partes: la primera se centra en algunas posiciones teóricas y las tradiciones con las que están asociadas; la segunda incorpora investigaciones y experiencias sobre procesos de apropiación de tecnologías; la tercera hace foco en las tecnologías en el ámbito educativo y la cuarta abre la discusión a los desafíos e interrogantes en 


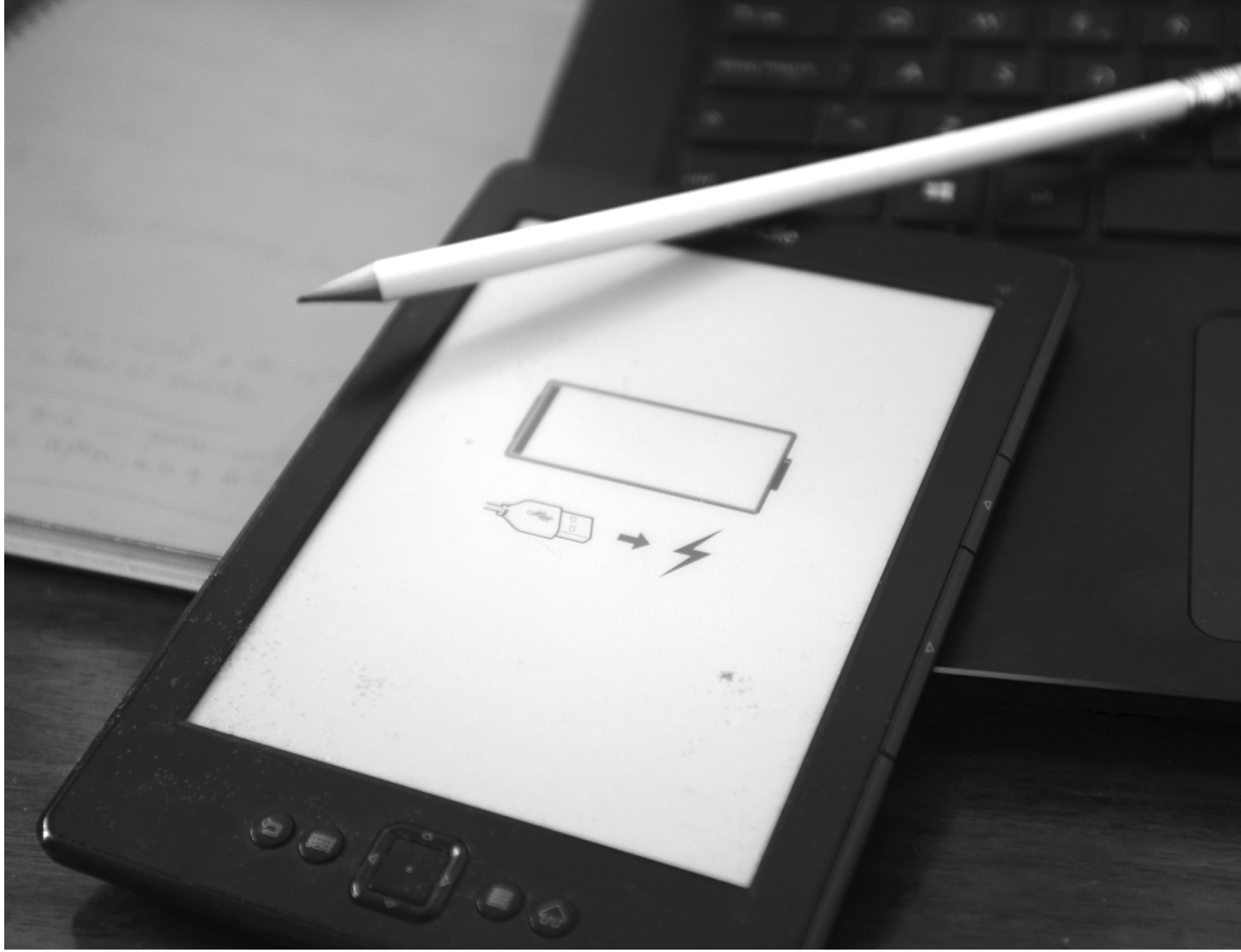

relación a la conjunción de tecnologías, ciudades y pantallas, lo que algunos ya denominan como Cuarta Revolución Industrial. A continuación, presento algunas ideas claves de los autores en cada parte del libro.

La primera parte, "Aproximaciones teóricas sobre la apropiación de tecnologías", construye un lugar teórico o posibles espacios de delimitación metodológica para abordar esta perspectiva, tanto en su dimensión práctica como simbólica y en campos como la política, el género o la educación, por nombrar algunos. Hay una marcada preocupación por crear un prisma analítico que permita ver algo más que una plataforma tecnológica desplegada para usuarios y gestionada por empresas y mercados. El objetivo principal de este apartado es profundizar en las múltiples capas del fenómeno de la apropiación, a partir de distintas miradas y un enfoque conectivo, interesado en reconstruir la socialidad teniendo en cuenta la matriz cultural y buscando un análisis integral para captar la complejidad y densidad de la apropiación tecnológica.

La idea de emancipación del espectador de Rancière, que Sandoval retoma en su trabajo para este libro (pp. 61-72), permite pensar la apropiación como distintas posibilidades de constituir formas de decir, maneras de ver y maneras de ser que rompan con aquellas impuestas por el orden de dominación; construir una capacidad colectiva de decir sobra la base de una transformación de nuestra relación con nuestra propia condición, a partir de la apropiación del sentido que habilita o se juega en las TIC.

La segunda parte, "Investigaciones y experiencias sobre procesos de apropiación de tecnologías", articula la teoría con el análisis empírico de varios casos de estudio 
que abordan, entre muchas otras, las siguientes preguntas: ¿Qué acciones se invocan para la apropiación? ¿De qué representaciones están dotadas? ¿A través de qué modelos de interacción participan? ¿Qué relación hay entre la población observada y la apropiación tecnológica? La perspectiva de análisis que ofrece es un importante acercamiento de nivel sociotécnico.

Las miradas que se aportan aquí arrojan luz sobre cómo funcionan las estructuras organizacionales de las plataformas tecnológicas, entendidas como espacios políticos e infraestructuras performativas, espacios que no son neutros y que permiten que "pasen cosas”. Las plataformas, tecnológicamente hablando, son proveedores de software, hardware y servicios que ayudan a codificar actividades sociales en una arquitectura computacional. Procesan (meta) datos mediante algoritmos y protocolos para luego presentar su lógica interpretada en forma de interfaces amigables con el usuario, que ofrecen configuraciones por defecto que reflejan las elecciones estratégicas de los propietarios de las plataformas. La incorporación de esas miradas al concepto de apropiación tecnológica permite avanzar con mayor profundidad en las plataformas como constructos tecnoculturales.

La tercera parte, "Tecnologías en el ámbito educativo", se encara desde distintas perspectivas y tiene en cuenta la complejidad que conlleva la intervención y adopción de las diversas tecnologías en el ámbito educativo. Decía Martín Barbero en 2002 que "la escuela está dejando de ser el único lugar de legitimación del saber, ya que hay una multiplicidad de saberes que circulan por otros canales, difusos y descentralizados” (p. 12). Sin dudas, esa diversificación y difusión del saber por fuera de la escuela es uno de los retos más fuertes que el mundo de la comunicación le plantea al sistema educativo. Algunos los llaman “saberes-mosaico" porque están hechos de trozos, de fragmentos, que sin embargo no impiden a los jóvenes, por ejemplo, tener un conocimiento más actualizado en física o en geografía que su propio maestro.

La escuela (o la educación formal) muchas veces no tiene una apertura a esos nuevos saberes, sino que más bien se pone a la defensiva y construye una idea negativa y moralista de todo lo que llega desde el ecosistema comunicativo de los medios y las tecnologías de comunicación e información. Pero ¿qué ocurre cuando el libro es desplazado como eje y centro de la cultura para los más jóvenes que ahora leen y escriben de otras maneras? Los trabajos en este apartado abordan directamente la relación entre tecnología y educación, y despliegan todo el abanico de desafíos y retos que comporta la mediación tecnológica en ese ámbito.

Por último, la cuarta parte, "Tecnologías, ciudades y pantallas: desafíos e interrogantes", nos interpela de cara al futuro. El desarrollo de la inteligencia artificial, el internet de las cosas, las ciudades inteligentes, nos plantean una pregunta: ¿De qué modo la revolución digital, internet y las redes sociales han transformado la sociedad y las relaciones? 0, mejor aún: ¿Cómo las seguirán transformando? El texto de Susana Finquelievich (pp. 219-236) da pistas para entender alguna de esas trasformaciones a partir de la intervención de la inteligencia artificial y cómo se reconfigura, por ejemplo, el mundo laboral a raíz de la llamada Cuarta Revolución Industrial.

El filósofo surcoreano Byung-Chul Han (citado en Geli, 2018) señala que el "dataísmo" es una característica de este siglo: "el hombre ya no es soberano de sí mismo, sino que es resultado de una operación algorítmica que lo domina sin que lo perciba”, una big data que nos sumerge en el infierno de lo igual. Pero también de lo 
desigual, como apuntan en su artículo Ester Schiavo y Alejandro Gelfuso (pp. 237-246) cuando explican el paradigma dominante de las ciudades inteligentes y la disputa material y simbólica por los sentidos de la apropiación de tecnologías digitales en las ciudades del siglo XXI.

Diego Levis (pp. 247-255) cierra el libro con un artículo que describe las amenazas de las pantallas y de la promesa cibernética que digitaliza la sociocultura, una característica del tecnocapitalismo contemporáneo, señala el autor, que se pregunta: ¿Cómo imaginar y construir un mundo diferente al que se va conformando en la sociedad de la pantalla al amparo del mito tecnodigital? Su pregunta y las referencias en su texto remiten a las ideas de Paul Ricoeur en Ideología y utopia (1989), ya que los discursos, tanto ideológicos como utópicos, son los lugares en los que la sociedad deposita las respuestas a los cambios históricos y sociales.

Crear un proyecto utópico significativo, como menciona Levis citando a Žižek, es una tarea que necesita del compromiso de todos, y este libro puede dar algunas pistas para comenzar (o continuar) ese proyecto.

\section{Referencias}

Geli, C. (7 de febrero de 2018). Byung-Chul Han "Ahora uno se explota a sí mismo y cree que está realizándose". El País. Recuperado de https://elpais.com/cultura/2018/02/07/actualidad/1517989873_086219.html

Lago Martínez, S., Álvarez, A., Gendler, M., y Méndez, A. (Coords.). (2018). Acerca de la apropiación de tecnologías: teoría, estudios y debates. Buenos Aires, Argentina: Ediciones del Gato Gris.

Martín-Barbero, J. (2002). Tecnicidades, identidades, alteridades: desubicaciones y opacidades de la comunicación en el nuevo siglo. Diálogos de la comunicación, 64, 8-23.

Ricoeur, P. (1989). Ideología y utopía. Barcelona, España: Gedisa.
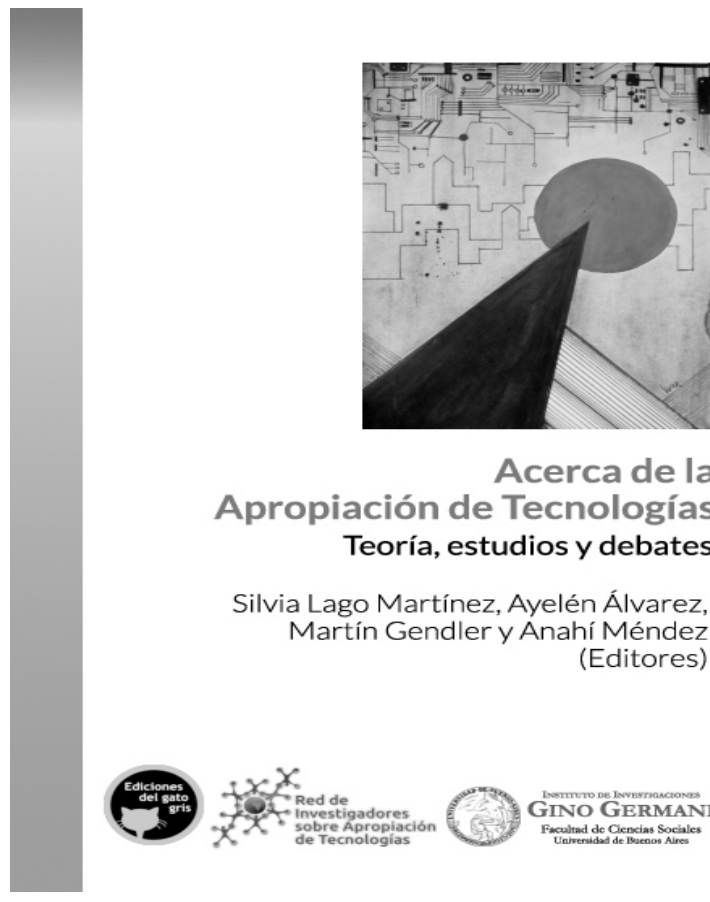

Acerca de la Apropiación de Tecnologías Teoría, estudios y debates

Silvia Lago Martínez, Ayelén Álvarez, Martín Gendlery Anahí Méndez (Editores)

\section{Ficha técnica}

Título: Acerca de la apropiación de tecnologías: teoría, estudios y debates.

Editores: Silvia Lago Martínez, Ayelén Álvarez, Martín Gendler y Anahí Méndez.

Autores: Guadalupe Álvarez; Fernando Andonegui;

Sheila Amado; Lucas Bang; Lorena Bassa; Walys Becerril Martínez; Erik Butrón Untiveros; Roxana Cabello; Silvina Casablancas; Delia Crovi Druetta; María Belén Fernández; Susana Finquelievich; Romina Gala; Alejandro Gelfuso; Alejo González López; Claudia Laudano; Diego Levis; Adrián López; Alejo González López; Susana Morales; María Julia Morales; Graciela Natanshon; Flavia Samaniego; Luis Sandoval; Ester Schiavo; Mónica Paz; Ana Rivoir.

Año de publicación: 2018.

Lugar de publicación: Ciudad autónoma de Buenos Aires, Argentina: Ediciones del Gato Gris.

Número de páginas: 255. 